\title{
INVARIANT MEANS AND FACTOR-SEMIGROUPS ${ }^{1}$
}

\section{CARROLL O. WILDE AND LOREN ARGABRIGHT}

1. Introduction. A left invariant mean on a discrete semigroup $S$ is a positive, normalized, left-translation-invariant linear functional on the Banach space $m(S)$ of all bounded, real-valued functions on $S$ under the supremum norm. It is well known [1] that if $S$ possesses a left invariant mean, then so does every homomorphic image of $S$; here we consider the problem of reversing this process.

The problem of lifting invariant means on quotients of a semigroup $S$ to $S$ itself was introduced by Dixmier [2]. Let $A$ be a distinguished subsemigroup of a semigroup $S$. (Define a relation $(r)$ on $S$ as follows: $s\left(r^{\prime}\right) t$ if and only if $s A \cap t A \neq \varnothing$, and $s(r) t$ if and only if there exist $u_{1}, u_{2}, \cdots, u_{n}$ in $S$ such that $s\left(r^{\prime}\right) u_{1}, u_{1}\left(r^{\prime}\right) u_{2}, \cdots, u_{n}\left(r^{\prime}\right) t$. A similar relation can be defined on the right, and $A$ is called distinguished if the two sets of classes coincide.) Dixmier showed that if $A$ and $S / A$ both possess left invariant means, then so does $S$.

In [3], E. Granirer observed that if $S$ is a semigroup in which each pair of right ideals has a nonvoid intersection, then the relation $(r)$ on $S$, defined by $s(r) t$ if and only if there exists $u \in S$ such that $s u=t u$, is a two-sidedly stable equivalence relation on $S$ and hence $S /(r)$ forms a semigroup under the natural multiplication [5]. The purpose of this paper is to prove that if $S /(r)$ has a left invariant mean, then so does $S$.

2. Notation and preliminaries. The only topology we consider in $m(S)^{*}$ is the $w^{*}$-topology. Let $q$ be the natural embedding of $S$ into $m(S)^{*}$, i.e., $q s(x)=x(s)$ for all $s \in S$ and $x \in m(S)$. Then the closure of $q S$ in $m(S)^{*}$ coincides with the Stone-Čech compactification, $\beta S$, of the discrete space $S$. If $A \subseteq S$, denote the closure of $q A$ in $\beta S$ by $\mathrm{Cl}(A)$.

Following Day [1], we define the Arens product of two means as follows. For $s \in S$ let $l_{s}$ be the left-translation operator in $m(S)$; i.e., $l_{s} x(t)=x(s t)$ for $t \in S$. The Arens product, $\mu \odot \nu$, of two means $\mu, \nu$ is defined on $m(S)$ by $\mu \odot \nu(x)=\mu\left(F_{\nu} x\right)$, where $F_{\nu} x(s)=\nu\left(l_{\varepsilon} x\right)$ for $s \in S$. The Arens product renders $\beta S$ and the set of all means on $m(S)$ semigroups, each containing $q S$ as a subsemigroup. A mean $\mu$ is left invariant if and only if $q s \odot \mu=\mu$ for all $s \in S$.

3. The main theorem. The purpose of this section is to prove our principal result:

Received by the editors May 27, 1966.

1 Research supported by National Science Foundation under Grant GP-4033. 
TheOREM. Let $S$ be a semigroup in which each pair of right ideals has a nonvoid intersection. Define a relation $(r)$ in $S$ by $s(r) t$ if and only if there exists $u \in S$ such that $s u=t u$; then $S /(r)$ is a semigroup. If $S /(r)$ has a left invariant mean, then so does $S$.

Through the proof $S$ and $(r)$ will be as described in the theorem.

LEMMA 1 (GRANIRER [3]). ( $r$ ) is an equivalence relation and is stable on both sides, i.e., $s(r) t$ implies $s u(r) t u$ and $u s(r) u t$ for $s, t, u$ in $S$; hence $S /(r)$ is a semigroup.

The proof used by Granirer will be illustrated in Lemma 2.

Lemma 2. Let $F$ be a finite subset of $S$ such that $s(r) t$ for all $s, t \in F$. Then there exists $u \in S$ such that Fu consists of one element.

Proof. Let $s(r) t, t(r) u$; take $v, w \in S$ such that $s v=t v, t w=u w$. Take $v^{\prime}, w^{\prime} \in S$ such that $v v^{\prime}=w w^{\prime}$; then $s v v^{\prime}=t v v^{\prime}=t w w^{\prime}=u w w^{\prime}=u v v^{\prime}$. The induction process has now been demonstrated.

First, T. Mitchell [6], then E. Granirer [4] showed that if $S /(r)$ consists of a single class, then $S$ has a left invariant mean which, in fact, lies in $\beta S$. In Lemma 3 we obtain points in $\beta S$ which, although not translation-invariant, are class-independent for each class.

Lemma 3. There exists a point $\omega$ in $\beta S$ such that $a(r) b$ in $S$ implies $q a \odot \omega=q b \odot \omega$.

Proof. Let $\left\{A_{1}, \cdots, A_{k}\right\}$ be a finite subset of $S /(r)$. Let $F_{i}$ be the family of all finite subsets of $A_{i}$ (as a set) for $i=1, \cdots, k$, each directed upward by inclusion, let $\mathcal{F}$ be the product directed set, and define a net over $\mathcal{F}$ as follows. If $F \in \mathcal{F}$, then $F=\left(F_{1}, \cdots, F_{k}\right)$ with $F_{i} \in \mathcal{F}_{i}$ for each $i$. Then there exist points $u_{i}, v_{i} \in S$ such that $F_{i} u_{i}$ $=\left\{v_{i}\right\}$ for $i=1, \cdots, k$. Since each finite collection of right ideals in $S$ has a nonvoid intersection, there exist points $w_{i} \in S$ such that $u_{1} w_{1}=\cdots=u_{k} w_{k}$; call this common product $s_{F}$. Then for each $i, F_{i} s_{F}=F_{i} u_{i} w_{i}=\left\{v_{i} w_{i}\right\}$; let $v_{i} w_{i}=t_{F}^{i}$. Let $\omega$ be a cluster point of the net $\left\{q s_{F}, F \in \mathcal{F}\right\}$, and let $\left\{q x_{n}, n \in N\right\}$ be a subset converging to $\omega$. We shall show that for each $i$ the net $\left\{q y_{n}^{i}, n \in N\right\}$ (here $x_{n}=s_{F_{n}}$, where $n \rightarrow F_{n}$ is the subnet function, and $y_{n}^{i}$ is the corresponding point $\left.t_{F_{n}}^{i}\right)$ converges, say to $\Psi_{i}$, in $\beta S$, and that $q a_{i} \odot \omega=\Psi_{i}$ for each $a_{i} \in A_{i}$. Fix any $a_{0}^{i} \in A_{i}$ and let $\Psi_{i}=q a_{0}^{i} \odot \omega$. Let $F_{0}=\left(\left\{a_{0}^{1}\right\}, \cdots,\left\{a_{0}^{\boldsymbol{k}}\right\}\right)$; by definition of subnet, there exists $n_{0} \in N$ such that $F_{n} \geqq F_{0}$ when $n \geqq n_{0}$ (i.e., $a_{0}^{i} \in F_{n}^{i}$ for each $i$, where $\left.F_{n}=\left(F_{n}^{1}, \cdots, F_{n}^{k}\right)\right)$. Thus $a_{0}^{i} x_{n}=y_{n}^{i}$ or $q a_{0}^{i} \odot q x_{n}=y_{n}^{i}$. Since $\lim _{n}\left(q a_{0}^{i} \odot q x_{n}\right)=q a_{0}^{i} \odot \omega=\Psi_{i}$ by continuity of the multiplication operator, we have $\lim _{n} y_{n}^{i}=\Psi_{i}$ for $i=1, \cdots, k$. For 
arbitrary $a_{i} \in A_{i}$, repeat the argument using subnets to obtain $q a_{i} \odot q x_{n}=q a_{0}^{i} \odot q x_{n}, i=1, \cdots, k$, for sufficiently large $n$. Then $\lim _{n}\left(q a_{i} \odot q x_{n}\right)=\Psi_{i}$; hence $q a_{i} \odot \omega=\Psi_{i}$ for each $i$. If we now let $T(A)$ for any $A \in S /(r)$ be the set of all $\omega \in \beta S$ which are class-independent for $A$, then each $T(A)$ is closed, and the above argument shows that the family $\{T(A): A \in S /(r)\}$ has the finite intersection property. The lemma now follows from compactness of $\beta S$.

Proof of MAIN THEOREm. Let $\omega \in \beta S$ be class-independent for each $A \in S /(r)$ by Lemma 3, and for each such $A$ let $\omega_{A}=q a \odot \omega$, where $a$ is any representative of $A$. Define a mapping $T$ on $m(S)$ to $m(S /(r))$ by $T x(B)=\omega_{B}(x)$. Then $T$ is positive, linear and preserves the constant functions. Let $\Psi$ be a left invariant mean on $m(S /(r))$ and define $\mu=T^{*} \Psi$; then $\mu$ is a mean on $m(S)$. If $s \in S$, then $\left[T\left(l_{s} x\right)\right](B)$ $=\omega_{B}\left(l_{s} x\right)=q s b \odot \omega(x)=\omega_{A B}(x)$, where $A$ is the class of $s$. Thus $\left[T\left(l_{s} x\right)\right](B)=T x(A B)=\lambda_{A} T x(B)$, where $\lambda$ is the left-translation operator in $m(S /(r))$. Now clearly $\mu\left(l_{s} x\right)=\mu(x)$.

\section{REFERENCES}

1. M. M. Day, Amenable semigroups, Illinois J. Math. 1 (1957), 509-544.

2. J. Dixmier, Les moyennes invariantes dans les semi-groups et leurs applications, Acta. Sci. Math. (Szeged) 12 (1950), 213-227.

3. E. E. Granirer, $A$ theorem on amenable semigroups, Trans. Amer. Math. Soc. 111 (1964), 367-379.

4. - Extremely amenable semigroups, Math. Scand. 17 (1965), 177-197.

5. E. S. Ljapin. Semigroups, Transl. Math. Monographs, Vol. 3, Amer. Math. Soc., Providence, R. I., 1963.

6. T. Mitchell, Fixed points and multiplicative left invariant means, Trans. Amer. Math. Soc. 122 (1966), 195-202.

University OF MinNesota 